\title{
Sextupole correction of the longitudinal transport of relativistic beams in dispersionless translating sections
}

\author{
R. J. England, J. B. Rosenzweig, G. Andonian, P. Musumeci, G. Travish, and R. Yoder \\ Department of Physics and Astronomy, University of California Los Angeles, Los Angeles, California 90095, USA
}

(Received 7 July 2004; published 31 January 2005)

\begin{abstract}
We examine the use of sextupole magnets to correct nonlinearities in the longitudinal phase space transformation of a relativistic beam of charged particles in a dispersionless translating section, or dogleg. Through heuristic analytical arguments and examples derived from recent experimental efforts, augmented by simulations using the particle tracking codes PARMELA and ELEGANT, sextupole corrections are found to be effective in optimizing the use of such structures for beam compression or for shaping the current profile of the beam, by manipulation of the second-order longitudinal dispersion. Recent experimental evidence of the use of sextupoles to manipulate second-order horizontal and longitudinal dispersion of the beam is presented. The theoretical and experimental results indicate that these manipulations can be used to create an electron bunch with a current profile having a long ramp followed by a sharp cutoff, which is optimal for driving large-amplitude wake fields in a plasma wake field accelerator.
\end{abstract}

DOI: 10.1103/PhysRevSTAB.8.012801

PACS numbers: 41.85.-p, 29.27.Eg, 41.75.Lx

\section{INTRODUCTION}

Several experiments [1-4] in the field of beam physics have recently been proposed that require, or may benefit from, the successful transport (with or without compression) of bunches of charged particles at large energy spread through a dispersionless translating section, or dogleg. This device, consisting of two consecutive bend magnets of opposite sense separated by dispersion-matching focusing optics, is commonly used in linear accelerator systems to translate the beam axis transversely, and it may also be used as a tool to compress or shape the current profile of a relativistic electron bunch. Examples of three such beam line structures are shown in Fig. 1.

For beams of large energy spread, this type of transport line tends to have a longitudinal (temporal) dispersion function with significant nonlinear contributions, i.e., containing higher than first order dependences in powers of the momentum error. The physical effect of these nonlinear contributions is to introduce quadratic and possibly higherorder correlations between energy and longitudinal position within the bunch. In applications that are sensitive to the beam's distribution in the longitudinal phase space, it is desirable to have the ability to manipulate these nonlinear effects. Such manipulations can be employed to shape the phase space distribution and thereby linearize the transport. In Sec. II we present several analytical results which help to illuminate these effects as well as their control using sextupole correction. In the interest of clarity and brevity, important derivations are reserved for the Appendices.

Our main results, contained in Secs. III and IV, consist of numerical and experimental studies pertaining to the control of nonlinear longitudinal effects in the dogleg sections on the linear accelerators at the UCLA-Neptune Laboratory and the ORION beam line at SLAC, shown in
Figs. 1(b) and 1(c). The goal of these studies is to shape the current profile of the beam in order to optimize it for use as a drive beam for the plasma wake field accelerator (PWFA). Simulations of the dogleg sections on these beam lines, presented in Sec. III, indicate that sextupole corrections could be implemented in order to linearize their longitudinal transport. With such transport, these doglegs can act as bunch compressors capable of producing electron bunches that rise linearly in density from head to tail, followed by a sharp drop. This type of longitudinal beam profile has been predicted to produce large-amplitude wake fields and high transformer ratios [5], making it ideal as a PWFA drive beam. In Sec. IV, we present new experimental results of horizontal dispersion and coherent transition radiation (CTR) bunch length measurements on the UCLA beam line of Fig. 1(b), which demonstrate the viability of using sextupoles to manipulate the bunch shape in a dogleg compressor.

Two additional applications, related to the visible to infrared SASE amplifier (VISA) Brookhaven Accelerator Test Facility (ATF) dogleg section of Fig. 1(a), are presented in Appendices C and D. In Appendix C, we discuss simulations of second-order nonlinear bunch compression, which have helped to explain the improved gain of the VISA self-amplified spontaneous emission free electron laser (SASE-FEL) when running the beam slightly offenergy, an effect which was observed but not fully understood during the first phase of that experiment [1]. In Appendix D, we discuss similar calculations for the currently ongoing second phase of the VISA experiment, which indicate that these second-order effects can be minimized using sextupole correction, in order to transport a beam though the dogleg section while preserving a strong linear momentum-time correlation (chirp). This beam, when delivered to the VISA undulator, may create a corresponding frequency chirp in the FEL output radiation 
TABLE I. Various parameter values for the dogleg sections of Fig. 1.

\begin{tabular}{lccccccccc}
\hline \hline Facility & $E(\mathrm{MeV})$ & $\rho(\mathrm{m})$ & $\theta(\mathrm{deg})$ & $\Delta s(\mathrm{~m})$ & $\varepsilon_{N}(\mu \mathrm{m})$ & $\sigma_{\delta}(\%)$ & $R_{56}(\mathrm{~m})$ & $T_{566}(\mathrm{~m})$ & $U_{5666}(\mathrm{~m})$ \\
\hline UCLA & 14 & 0.30 & 45 & 2 & 5 & 1.7 & 0.05 & 2.54 \\
VISA-I & 71 & 1.15 & 20 & 15 & 3.7 & 1.02 & -0.0045 & -10.1 \\
VISA-II & 71 & 1.15 & 20 & 15 & 2.42 & 0.56 & -0.016 & -7.17 & 172.5 \\
ORION & 55 & 0.84 & 22 & 14 & 4.4 & 2.2 & -0.014 & -1.8 & 29.7 \\
\hline \hline
\end{tabular}

pulse [2]. As a point of reference, values for various parameters used in the discussions of Sec. II, including the beam energy $E$, the bend radius $\rho$, bend angle $\theta$, total path length $\Delta s$, normalized emittance $\varepsilon_{N}$, rms momentum spread $\sigma_{\delta}$, and the transport matrix elements $R_{56}, T_{566}$, and $U_{5666}$ (without sextupole correction) are given in Table I for the dogleg sections on each of these beam lines. The meanings of these matrices are explained in Sec. II.

\section{BACKGROUND AND HEURISTIC ANALYSIS}

The transformation of the $6 \mathrm{D}$ trace space vector $\boldsymbol{X}=$ $\left(x, x^{\prime}, y, y^{\prime}, z, \delta\right)$ of a beam produced by a system of magnetic elements can be represented to arbitrary order in a power series expansion of the trace space coordinates. This method was originally developed for use in the particle tracking code TRANSPORT [6]. In component form, the transformation from the initial to final coordinates $\left(\boldsymbol{X}_{0} \rightarrow\right.$ $\boldsymbol{X}_{f}$ ) reads

$$
\begin{aligned}
\left(\boldsymbol{X}_{f}\right)_{i}= & R_{i j}\left(\boldsymbol{X}_{0}\right)_{j}+T_{i j k}\left(\boldsymbol{X}_{0}\right)_{j}\left(\boldsymbol{X}_{0}\right)_{k} \\
& +U_{i j k l}\left(\boldsymbol{X}_{0}\right)_{j}\left(\boldsymbol{X}_{0}\right)_{k}\left(\boldsymbol{X}_{0}\right)_{l}+\cdots,
\end{aligned}
$$

where $R_{i j}, T_{i j k}, U_{i j k l}, \ldots$ are transport matrices (or tensors) of increasing order, and there is an implied summation on repeated indices. Since we are concerned with the transformation of the longitudinal phase space of the beam, we will focus upon the $z$ or $i=5$ component of Eq. (1), which has the general form $z_{f}=z_{0}+[$ chromatic terms $]+$ [geometrical terms]. For beams of small transverse emittance but with a large energy spread, the chromatic terms in the transformation will tend to dominate the final form of the longitudinal profile. Among these, the strongest contributors are the longitudinal dispersion terms, which are dependent on the momentum; the coupling of final longitudinal position to the initial transverse coordinates is relatively quite small. Hence, we may formulate the following approximation, including terms up to third order in the momentum error $\delta$,

$$
z_{f} \cong z_{0}+R_{56} \delta+T_{566} \delta^{2}+U_{5666} \delta^{3}+\cdots .
$$

The first order coefficient $R_{56} \equiv\left(\partial z_{f} / \partial \delta\right)_{\delta \rightarrow 0}$ represents the longitudinal dispersion function. It is proportional to the negative of the temporal dispersion function, sometimes denoted by $\eta_{\tau}$. The remaining elements, $T_{566}, U_{5666}, \ldots$, are higher-order momentum-error contributions to the longitudinal dispersion. We may consider Eq. (2) to apply to a beam of small transverse emittances and large energy spread. ${ }^{1}$ It is also presumed that the beam is sufficiently relativistic that space charge may be neglected. The point at which higher-order terms in Eq. (2) may be truncated depends upon the energy spread of the beam. In practice it is rarely necessary to consider higher than third-order contributions for single-pass transport.

For a dogleg structure in which the quadrupoles have been successfully used to eliminate the linear horizontal dispersion and its derivative with respect to path length $\left(\eta_{x}, \eta_{x}^{\prime} \rightarrow 0\right)$, the analytical forms of the first- and secondorder terms may then be expressed as follows:

$$
\begin{gathered}
R_{56}=\frac{\Delta s}{\gamma_{0}^{2}}-2 \rho(\theta-\sin \theta), \\
T_{566}=4 \rho \sin ^{2}(\theta / 2) \cos (\theta / 2)+\sum_{W} a_{i 6 k} T_{i 6 k} .
\end{gathered}
$$

Here $\rho$ is the bend radius, $\theta$ is the bend angle, $\Delta s$ is the total path length (including the bends), and $\gamma_{0}$ is the central energy of the beam. The summation in the second expression is over the set $W$ of values of $i, k$ corresponding to the nonzero transverse second-order chromatic terms,

$$
\begin{aligned}
W & =\{(i, k):(i, k) \\
& =(1,1),(1,2),(1,6),(2,1),(2,2),(2,6),(5,1),(5,2)\},
\end{aligned}
$$

and $a_{i 6 k}$ are the corresponding coefficients, which are functions of $\theta$ and $\rho$. For the sake of space, we will relegate the explicit forms of these functions to Appendix A, where Eqs. (3) and (4) are derived.

In view of Eq. (4) and by virtue of the same argument by which the coupled transverse-chromatic terms were ignored in Eq. (2), we expect the dominant dependence of the second-order longitudinal dispersion term $T_{566}$ to derive from the second-order horizontal dispersion terms $T_{166}$ and $T_{266}$. Sextupole magnets are the obvious candidate for eliminating this sort of nonlinear effect, as they are inherently second order and are routinely used for making chromatic corrections to $T_{166}$ and $T_{266}$ in bending transport.

Since the second-order transport matrix for a pure sextupole contains only geometrical terms, coupling to $T_{566}$ is accomplished by placing the sextupoles in a region of large horizontal dispersion. The sextupole field strength then

\footnotetext{
${ }^{1}$ The zero emittance claim is false. One may launch a point beam with finite angular spread (zero emittance) which demands use of other terms in Eq. (2).
} 
couples to the $x$ coordinate, which affects $T_{566}$ via the firstand second-order horizontal dispersion, $R_{16}$ and $T_{166}$. If we assume that the arrangement of quadrupoles and drifts between the two dipole magnets is symmetric about the midpoint of the dogleg and include two symmetrically placed sextupole magnets whose geometrical field strengths are $\kappa$ and $\alpha \kappa$, respectively, then Eq. (5) is found to assume a linear dependence upon the sextupole field strength of the form

$$
T_{566}(\kappa)=A-C(1-\alpha) \kappa,
$$

where $A$ and $C$ are algebraic functions of $\theta$ and $\rho$, as well as the drift lengths and quadrupole focal lengths. These functions and the derivation of Eq. (6) are found in Appendix A.

If the goal is to eliminate $T_{566}$ altogether, then (i) to avoid asymptotic behavior, the value of $\alpha$ (the ratio of the two sextupole field strengths) should not approach unity, and (ii) in order to minimize $\kappa$ the quantity $C(1-\alpha)$ should be large and therefore $\alpha$ should be negative. A simple choice in agreement with these requirements is $\alpha=$ -1 , corresponding to sextupole fields equal in magnitude but of opposite polarity. As a rule, the minimum number of sextupoles needed is equal to the number of second-order matrix elements one wishes to eliminate. Therefore $\alpha=0$ is also a possibility, although the elimination of one sextupole would disrupt the optical symmetry and would require the surviving one to have twice the field strength. Minimization of the required sextupole fields, through appropriate placement of the correcting magnets, is desirable from the standpoint of preventing the inadvertent introduction of strong second-order geometrical effects, as well as third-order chromatic effects.

The sextupole correction of $T_{566}$ in this system often has the added effect of minimizing the horizontal emittance growth, due to the coupling of $T_{566}$ to the second-order horizontal dispersion discussed above. For a beam of large energy spread and small transverse emittance, the nonlinear emittance growth is dominated by the second-order horizontal dispersion elements $T_{166}$ and $T_{266}$. The final rms emittance is then approximately (see Appendix B)

$$
\varepsilon_{x, f} \cong \sqrt{\operatorname{det}\left[M_{x} \Sigma_{x, 0} M_{x}^{T}+\sigma_{\delta}^{2} \boldsymbol{d} \boldsymbol{d}^{T}+3 \sigma_{\delta}^{4} \boldsymbol{D} \bar{D}^{T}\right]},
$$

where $\boldsymbol{d}$ and $\boldsymbol{D}$ are the first- and second-order horizontal dispersion vectors, respectively, $\sigma_{\delta}=\left\langle\delta^{2}\right\rangle^{1 / 2}$ is the rms momentum spread, $M_{x}$ is the $2 \times 2$ linear transport matrix for the $\left(x, x^{\prime}\right)$ trace space plane, and $\Sigma_{x, 0}$ is the corresponding initial matrix of second moments, i.e.,

$$
\begin{aligned}
& \boldsymbol{d} \equiv\left(\begin{array}{l}
R_{16} \\
R_{26}
\end{array}\right), \quad \boldsymbol{D} \equiv\left(\begin{array}{l}
T_{166} \\
T_{266}
\end{array}\right), \quad M_{x}=\left(\begin{array}{ll}
R_{11} & R_{12} \\
R_{21} & R_{22}
\end{array}\right), \\
& \Sigma_{x, 0}=\left(\begin{array}{cc}
\left\langle x_{0}^{2}\right\rangle & \left\langle x_{0} x_{0}^{\prime}\right\rangle \\
\left\langle x_{0} x_{0}^{\prime}\right\rangle & \left\langle x_{0}^{\prime 2}\right\rangle
\end{array}\right) .
\end{aligned}
$$

The first of the three terms inside the determinant in Eq. (7) is the contribution from the initial emittance, which would be invariant if the transformation were governed solely by the linear matrix $M_{x}$. Consequently, if the first order horizontal dispersion and its derivative are eliminated in accordance with the discussion surrounding Eqs. (3) and (4), then $\boldsymbol{d} \rightarrow 0$ and the emittance growth described by Eq. (7) is dominated by the third term in square brackets. The coupling of longitudinal to horizontal dispersion is such that for the sextupole configuration described above the values of $T_{166}$ and $T_{266}$ tend to be reduced under the sextupole correction of $T_{566}$. Consequently, in many cases sextupole correction of longitudinal dispersion also has a reducing effect upon the transverse emittance.

\section{SIMULATION RESULTS}

The primary focus of our study is to test the concept of creating a beam that has a relatively long (many picoseconds) rising current profile, followed by a short (subpicosecond) fall time. The dogleg section on the beam line at the UCLA-Neptune Advanced Accelerator Laboratory, shown in Fig. 1(b), has been designed and constructed with this goal in mind [3]. Sextupole corrections on the dogleg section, shown in Fig. 1(c), of the proposed low-energy beam line for the ORION facility at Stanford Linear Accelerator Center (SLAC) have also been considered for the same purpose, namely, the creation of a ramped current profile [4].

This type of profile is of considerable interest as a driver for the PWFA, as it allows for a high transformer ratio (i.e., the ratio of the peak accelerating field found in the wake to the peak decelerating field experienced by the driving beam). We discuss below the ramped beam experiment at UCLA-Neptune, and the ORION project at SLAC, which we analyze with simulations using the tracking codes ELEGANT [7] and PARMELA [8].

\section{A. Optimal current profile for the PWFA drive beam}

Because of their capacity to support large electric fields, plasmas have been considered in recent years as a means for acceleration of charged particles capable of producing field gradients larger than those achievable with traditional radio frequency linear accelerating cavities by several orders of magnitude. Longitudinal field gradients well in excess of $1 \mathrm{GeV} / \mathrm{m}$ can be obtained by the excitation of large-amplitude relativistic waves in a plasma. Various acceleration schemes have been proposed which rely upon driving such plasma waves, using either a short intense laser beam [laser wake field accelerator (LWFA)] or a short relativistic electron beam PWFA [9-12]. In the case of the PWFA, the transformer ratio (the maximum longitudinal accelerating electric field in the wake of the driving beam divided by the maximum decelerating field within the beam) is a figure of merit which provides a measure of the maximum energy gain of a test charge injected behind the bunch. 
(a)

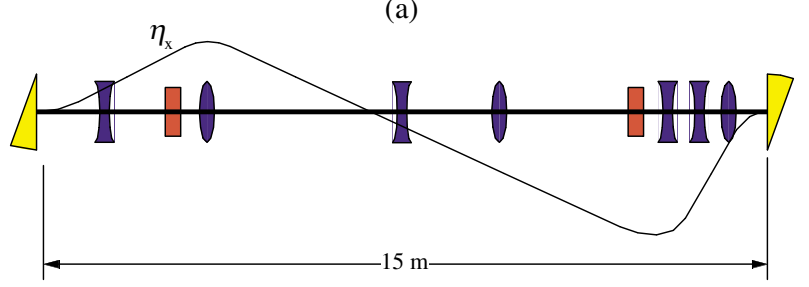

(b)

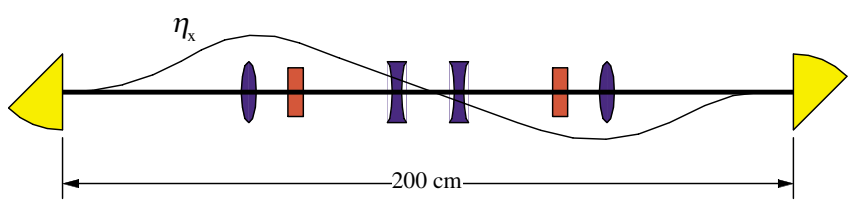

(c)

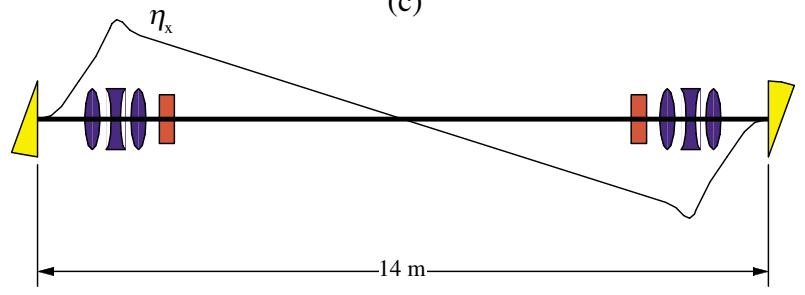

FIG. 1. (Color) Cartoon drawings of the dispersionless translating sections at (a) ATF-VISA, (b) UCLA-Neptune, and (c) ORION-SLAC. Each drawing is scaled to fit the figure. Wedges, blue lenses, and red rectangles represent dipoles, quadrupoles, and sextupoles, respectively. In each drawing an approximate representation of the horizontal dispersion function $\eta_{x}$ is superimposed.

For a driving bunch with a symmetric current profile and finite length, the value of the transformer ratio can be shown to always be less than two [13,14]. Various methods have been proposed to overcome this limitation, the most promising of which include the use of a single asymmetric drive bunch [15] or a ramped bunch train [16]. In the case of a single asymmetric drive bunch a "doorstep" profile (i.e., a square pulse for the first quarter of a plasma period, followed by a triangular ramp) approximates the optimal asymmetric current distribution that maximizes the transformer ratio and forces the retarding potential to be constant within the bunch. The analytically derived transformer ratio of such a beam is found to be $R=k_{p} L$, where $L$ is the length of the bunch and $k_{p}=\omega_{p} / c$ is the inverse plasma skin depth [5]. For such a profile $R$ may therefore exceed two so long as the bunch is longer than two plasma skin depths.

An example of the longitudinal trace space distribution of such a beam, artificially created from an idealized linear transformation (considering only $R_{56}$ ), characteristic of a dogleg applied to a beam distribution at the exit of a photoinjector electron source, as simulated by the beam modeling code PARMELA, is shown in Fig. 2(a). In Fig. 2(b) the corresponding current profile (in red) is superimposed with an ideal doorstep ramped profile (in black) discussed above. A two-dimensional particle-in-cell (PIC) simulation of the longitudinal wake field excited by this high-charge beam in a plasma of density $10^{16} \mathrm{~cm}^{-3}$, shown in Fig. 2(c), predicts a peak field of $10 \mathrm{GV} / \mathrm{m}$ with a transformer ratio of 11 .

\section{B. Beam shaping and compression at UCLA-Neptune and ORION}

As was discussed in the Introduction, a scheme has been recently proposed [3] for the creation of a beam which approximates the asymmetric ramped current profile, using first- and second-order beam optics in a dogleg compressor. The proposed method takes advantage of the rf curvature in the longitudinal phase space distribution of a positively chirped (i.e., back-of-crest) driving beam. Under a pure negative $R_{56}$ compression of the (a)

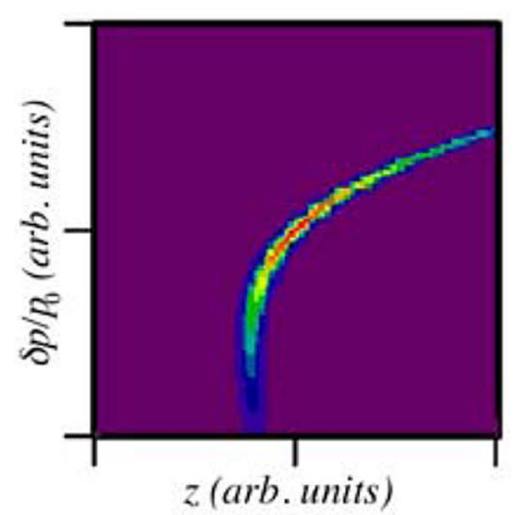

(b)

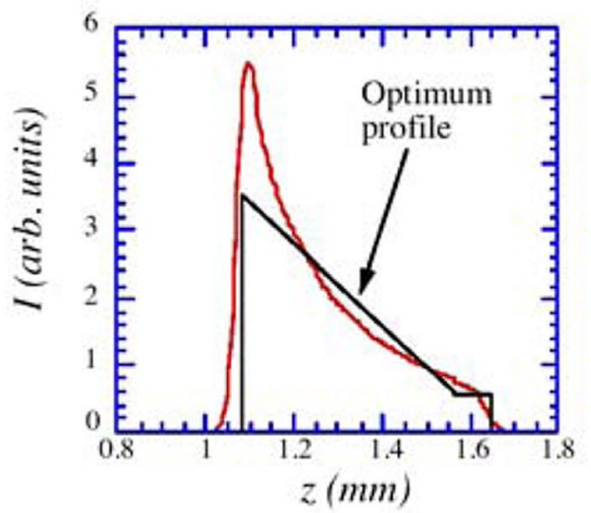

(c)

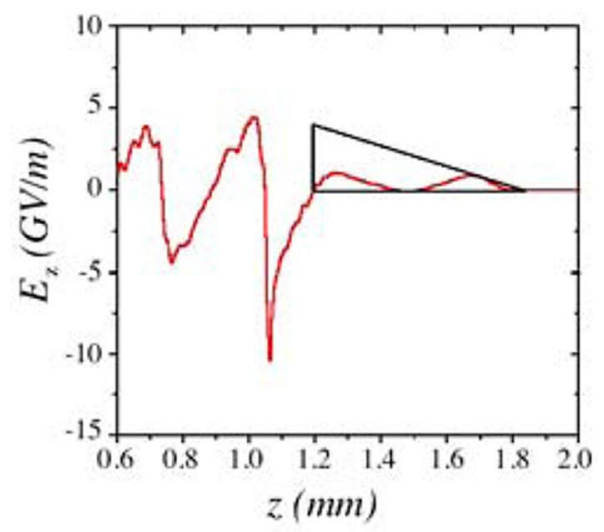

FIG. 2. (Color) Plot showing the longitudinal phase space (a) and density profile (b) of a ramped beam produced by linear dogleg (negative $R_{56}$ ) compression, as well as a PIC simulation (c) of the wake field produced by such a beam in a plasma of density $2 \times 10^{16} \mathrm{~cm}^{-3}$, with $6 \mathrm{nC}$ charge. 
(a)
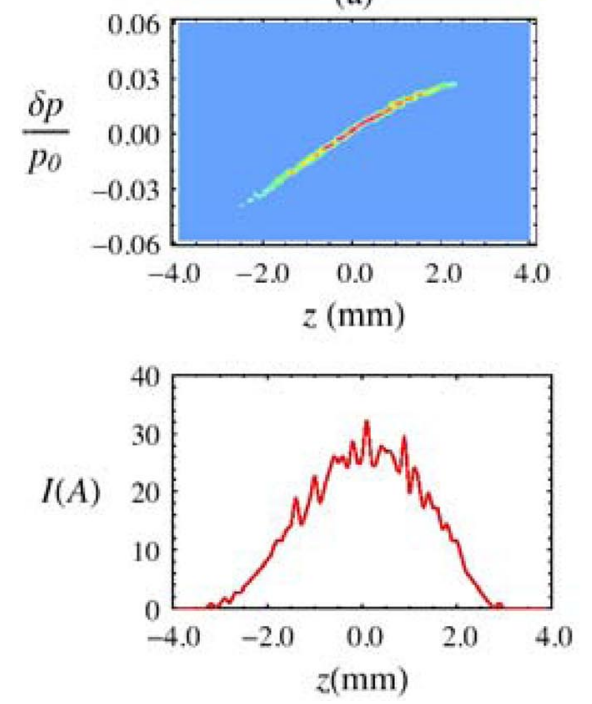

(b)
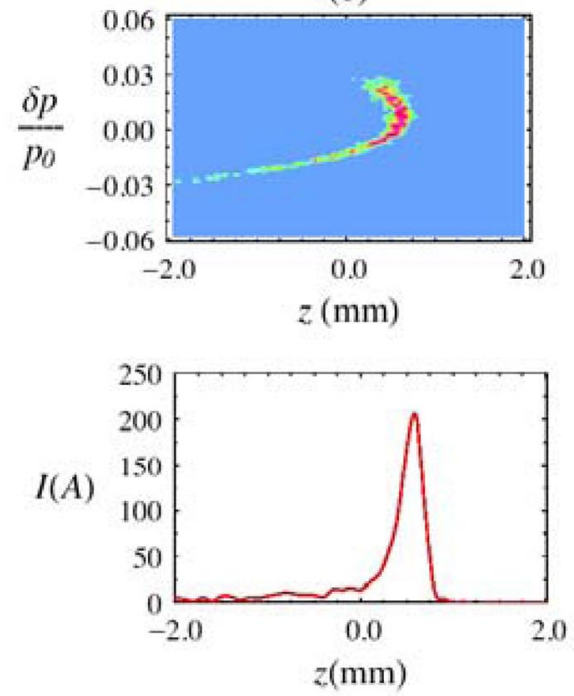

(c)
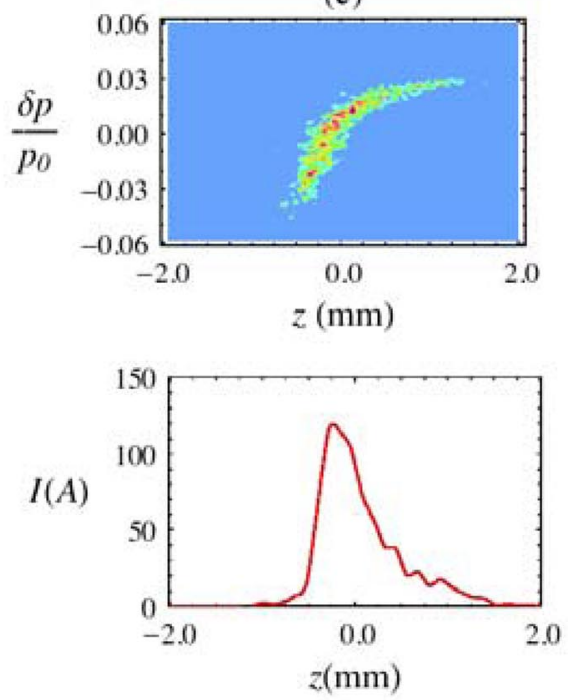

FIG. 3. (Color) Plots of the $z$ trace space and current profile from PARMELA and ELEGANT simulations of the UCLA accelerator beam line showing (a) the beam at the entrance of the dogleg compressor, and the same beam at the end (b) without sextupole correction and (c) with sextupole correction.

longitudinal phase space, such a distribution results in a ramp-shaped current profile of a few picosecond to subpicosecond duration, which is ideal for use as a driving beam for large-amplitude plasma wake fields with high transformer ratios.

A proof-of-principle experiment is currently underway at the UCLA-Neptune linear accelerator laboratory, using the dogleg section shown in Fig. 1(b) as a negative $R_{56}$ compressor. A PARMELA simulation of the beam at the entrance to the compressor in Fig. 3(a) shows the characteristic chirp in momentum and the rf curvature imposed by the accelerating structure.

The longitudinal phase space distributions at the exit of the compressor, predicted from simulations using the matrix-based transport code ELEGANT, are shown without and with sextupole correction in Figs. 3(b) and 3(c), respectively. The S-shaped distribution in Fig. 3(b) is evidence of the quadratic momentum dependence of the $z$ transformation produced by the second-order $T_{566}$ contribution in Eq. (2). When sextupole fields are utilized in accordance with the description of Sec. II to eliminate this contribution, the resulting distribution [Fig. 3(c)] is found to correspond very closely to that produced by a linear $R_{56}$ transformation, such as the one in Fig. 2. The resulting current profile exhibits a sharp drop in current at the back of the bunch, where the distribution begins to turn over on itself, preceded by an approximately linear ramp of the sort described in [5] as being ideal for generating large transformer ratios in a wake field accelerator.

It has been observed recently [17] that space-charge driven transverse phase space bifurcation and accompanying emittance growth are potential hazards encountered in low-energy (12-14 MeV) compression at Neptune. To gauge the transverse effects arising separately from non- linearities and space-charge forces, ELEGANT and PARMELA simulations were employed to calculate the normalized transverse emittance $\varepsilon_{n, x} \equiv(\beta \gamma)_{0} \varepsilon_{x}$ of the beam. The ELEGANT simulation, with sextupoles turned off, predicts an emittance growth in the Neptune dogleg due to nonlinear effects of $\Delta \varepsilon_{n, x}=13 \mathrm{~mm}$ mrad over the initial value of $5 \mathrm{~mm}$ mrad at the entrance. This is consistent with the approximation of Eq. (7), which gives $\Delta \varepsilon_{n, x} \approx$ $12 \mathrm{~mm}$ mrad.

With sextupoles turned on, ELEGANT predicts a much improved $\Delta \varepsilon_{n, x}=1.7 \mathrm{~mm} \mathrm{mrad}$, due to partial cancellation of the $T_{166}$ and $T_{266}$, as discussed in Sec. II. To gauge the effect of space-charge velocity field forces in the dogleg compressor, a calculational model for sextupoles was introduced into the PARMELA source code and simulations were run using PARMELA's point-to-point space-charge routine. With the space-charge routine turned off, the PARMELA results match the ELEGANT prediction of $\Delta \varepsilon_{n, x}=$ $1.7 \mathrm{~mm}$ mrad. With the space-charge routine turned on, PARMELA predicts a total emittance growth of $\Delta \varepsilon_{n, x}=$ $11.6 \mathrm{~mm} \mathrm{mrad}$, for a $300 \mathrm{pC}$ beam, indicating a significant additional contribution due to space-charge forces. These results lie in the intermediate range of $\Delta \varepsilon_{n, x}$ values measured in [17] and do not show evidence of the sort of phase space bifurcation reported there. The predicted growth in transverse emittance, however, imposes restrictions upon the focusability of the beam, requiring sharper focusing angles and higher gradient quadrupole magnets in order to, for example, match the beam into a PWFA. To meet these concerns, a high-gradient $(100 \mathrm{~T} / \mathrm{m})$ focusing system is being developed using permanent magnet quadrupoles for use after the Neptune dogleg.

This beam-shaping scheme has also been proposed [4] for future implementation on the dogleg leading from the 

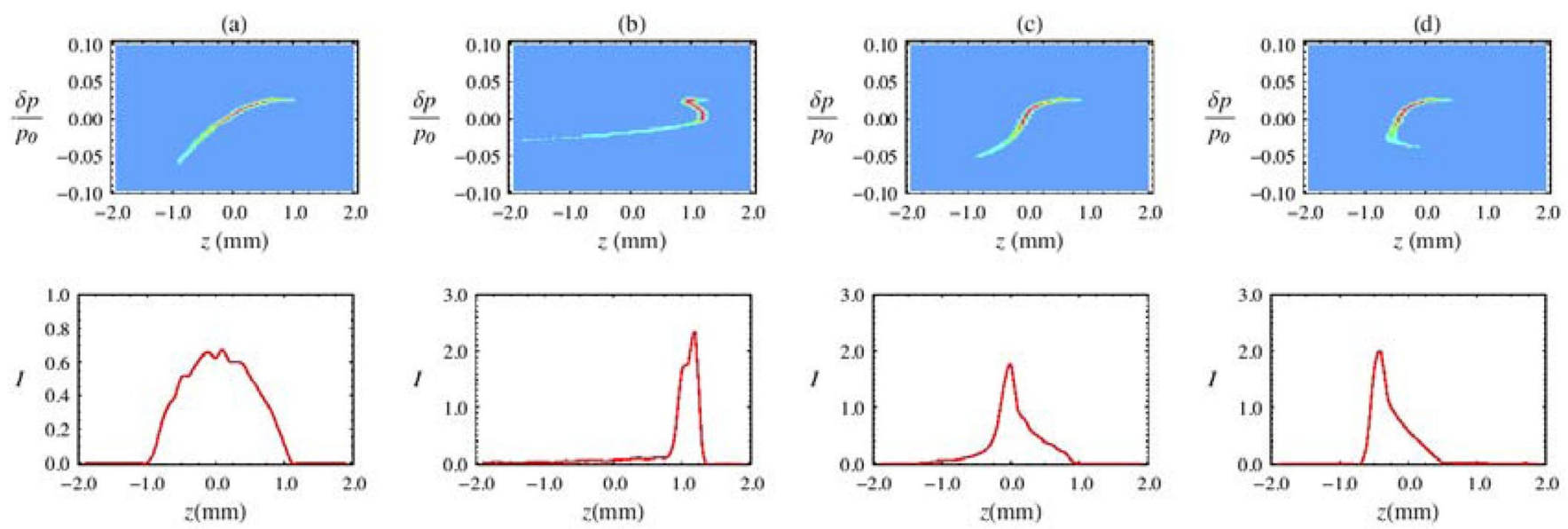

FIG. 4. (Color) Plots of the $z$ trace space and current profiles from PARMELA and ELEGANT simulations of the ORION dogleg section showing (a) the beam at the entrance, and the same beam at the end (b) without sextupole correction and (c) with sextupole correction. In (d) symmetrically placed octupoles are inserted for third-order correction. Beam currents are in arbitrary units, but the scale is the same on all plots.

main transport line to the low-energy $(50 \mathrm{MeV})$ experimental section of the ORION beam line at the Stanford Linear Accelerator, shown schematically in Fig. 1(c). Here the goal is again that of providing a venue for PWFA experiments using ramped bunches. The symmetrical placement of a pair of quadrupole triplets at the extreme ends of the structure is due to the spatial constraint imposed by a wall through which the middle section of the beam line passes. This quadrupole configuration, when compared with a more conventional arrangement such as that of Fig. 1(b), is found to result in $T_{566}$ values which are larger by about $45 \%$ and therefore require stronger compensating fields in the sextupoles. However, other significant nonlinear effects $\left(T_{266}, T_{166}, T_{561}\right.$, and $\left.T_{562}\right)$ are found to be reduced by this geometry, offsetting the danger of amplifying such terms by the use of stronger sextupoles.

Of more critical concern is the prediction of strong thirdorder effects in ELEGANT simulation results, primarily $U_{5666}$, due in part to the large $(2.2 \%$ rms, nearly $9 \%$ full) momentum spread found in this scenario. The momentum spread is enhanced at ORION with respect to Neptune and ATF (all three have similar $S$-band photoinjectors), by use of $X$-band postacceleration linacs. The large third-order chromatic effect arising from $U_{5666}$ is shown in the simulation results of Fig. 4, in which a chirped beam (a) with a $2.2 \%$ rms energy spread is injected into the ORION dogleg section producing at its exit the final distributions (b) without sextupole correction and (c) with sextupole correction. Qualitative comparison reveals in the corrected distribution in Fig. 4(c) the presence of a low-energy "tail" which is not observed, for example, in the ramped distribution of Fig. 3(c). Examination of the extra component to the transformation exhibited by this tail reveals it to be primarily third order and to possess a dominant component that is cubic in the momentum error, corresponding to the term $U_{5666}$ in Eq. (2). This effect may be compensated to some degree by the insertion of symmetrically positioned octupole magnets outside of the focusing triplets. However, the resulting collusion of second- and third-order effects distorts the current profile and destroys the desired hard edged cutoff at the back of the beam. As shown in Fig. 4(d), overcompensating with the octupoles can restore this hard edge but results in a new low-energy tail extending in the forward (positive $z$ ) direction. A full treatment of these effects requires an examination of third-order effects, which we reserve for future study.

\section{RECENT EXPERIMENTAL RESULTS}

Initial investigations of the effects of sextupole correction on the dogleg beam line of Fig. 1(b), dubbed S-Bahn, ${ }^{2}$ have been conducted at the UCLA-Neptune Laboratory. A more detailed diagram of this beam line is shown in Fig. 5. The recent experiments include measurements of the horizontal dispersion to second order in momentum error and CTR interferometry bunch length measurements. Both types of measurements were performed parametrically as functions of the sextupole field strengths.

The linear horizontal dispersion function $\eta_{x}$ (or $R_{16}$ ) was minimized in accordance with the discussion surrounding Eqs. (3) and (4) by adjusting the quadrupoles to reduce horizontal deflection of the beam centroid at the midpoint of the dogleg (Screen 11 in Fig. 5) with respect to a fractional perturbation $\zeta$ of the field strengths of all quadrupoles and dipoles on the dogleg section (labeled B1, Q1, Q2, and B2). The centroid deflection $\Delta x_{\text {cen }}$ under these conditions is equivalent to that which would be experienced by an off-momentum particle with momentum

\footnotetext{
${ }^{2}$ After a train system in Hamburg, Germany, and in honor of its geometry.
} 


\section{S-BAHN}

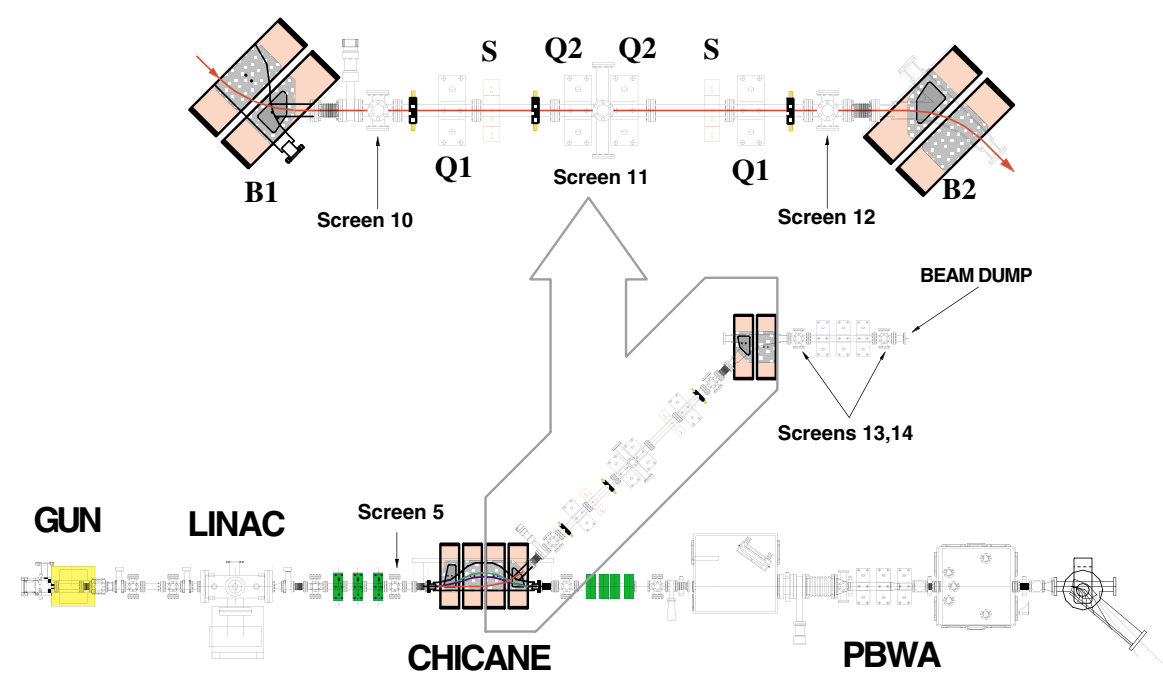

FIG. 5. (Color) Schematic of the linear accelerator beam line at the UCLA-Neptune Laboratory, with a blowup of the S-Bahn dogleg section.

error $-\zeta$, and is given to second order in powers of $\zeta$ by

$$
\Delta x_{\mathrm{cen}}=-R_{16} \zeta+T_{166} \zeta^{2}+O\left(\zeta^{3}\right)
$$

Consequently, the first- and second-order horizontal dispersion terms $R_{16}$ and $T_{166}$ can be obtained empirically by fitting the measured centroid deflection to a quadratic in $\zeta$. Empirical values of $T_{166}$ at the location of Screen 13 in Fig. 5 were obtained using this method, and are compared in Table II with the ELEGANT simulation predictions for three different configurations of the sextupole fields. The geometrical field strength $\kappa$ and ratio $\alpha$ correspond with the quantities in Eq. (6).

Experimental errors in Table II correspond to a 95\% confidence level. Since the quadrupoles were set to eliminate the linear dispersion, $R_{16}$ in all three cases was found to be zero to within the experimental error. Measurements of the rms beam size on Screens 5, 10, 11, 12, 13, and 14 agree with the ELEGANT simulation results to within $20 \%$.

Since the horizontal dispersion does not provide a diagnostic of the longitudinal trace space, the measurements of Table II were performed using a beam with no momentum chirp and a relatively small $(0.5 \%)$ energy spread. To obtain information about the effect of the sextupoles on the longitudinal distribution of the beam, the beam was

TABLE II. Comparison of experimental and simulated second-order horizontal dispersion values for various sextupole field settings.

\begin{tabular}{rrrr}
\hline \hline$\kappa\left(\mathrm{m}^{2}\right)$ & \multicolumn{1}{c}{$\alpha$} & \multicolumn{1}{c}{$T_{166, \exp }(\mathrm{m})$} & $T_{166, \text { sim }}(\mathrm{m})$ \\
\hline 0 & 0.00 & $2.56 \pm 0.59$ & 2.54 \\
537 & -2.13 & $0.22 \pm 0.77$ & 0.26 \\
995 & -1.55 & $-1.27 \pm 0.93$ & -1.69 \\
\hline \hline
\end{tabular}

then chirped in momentum by injecting it with an $\mathrm{rf}$ phase offset of $-28^{\circ}$ relative to the crest of the accelerating field in the standing wave linac cavity. The bunch length was then measured at different sextupole settings using CTR autocorrelation. Transition radiation emitted by the beam at a metal foil on Screen 14 of Fig. 5, oriented at $45^{\circ}$ incidence, was autocorrelated using a Martin-Puplett-type interferometer with wire grid polarizing beam splitters [18]. The bunch length $\sigma_{t}$ was extracted from the interferograms using the time-domain fitting procedure of Ref. [19]. The extracted values are plotted in Fig. 6 as a function of sextupole field strength $\kappa$. The ratio of the two sextupole fields was set to $\alpha=-1$. The data show the dependence of bunch length upon the magnitude of the sextupole correction, with an approximately twofold compression occurring near the field value $\kappa=1094 \mathrm{~m}^{-3}$.

It should be noted that, due to both the limited frequency bandwidth of the autocorrelator apparatus and the nature of the fitting procedure used to extract the pulse length from the data (which assumes a Gaussian current profile), for a beam whose temporal profile is asymmetric, the value of $\sigma_{t}$ obtained from the interferogram is more closely connected with the FWHM than with the rms width of the distribution. Consequently, we have found that obtaining a theoretical prediction to complement the data of Fig. 6 involves a somewhat complicated computational procedure, the final result of which is superimposed as a dashed curve.

To produce this theoretical curve, first the creation and transport of the beam in the accelerating section were simulated using the tracking code PARMELA. This detailed simulation employed 5000 macroparticles, whose initial temporal profile (inherited from the laser pulse) was modulated in a way consistent with observations of the energy 


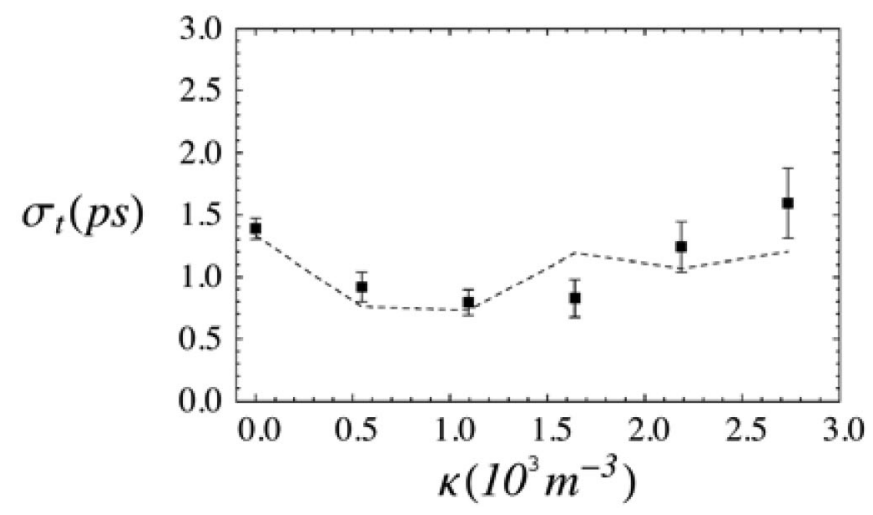

FIG. 6. CTR autocorrelator measurements of electron bunch length a as a function of sextupole field strength, with superimposed theoretical result (dashed line) obtained from PARMELA/ ELEGANT simulation combined with an autocorrelation algorithm.

modulation of the beam, and a $-28^{\circ}$ phase offset in the linac, producing a chirped beam. The set of output $6 \mathrm{D}$ trace space coordinates obtained from PARMELA was then used as the input beam for an ELEGANT simulation of the dogleg section, including a truncation of outlying particles consistent with the observed $60 \%$ electron transmission efficiency through the device. The longitudinal $(z)$ coordinates of the particles were extracted from the ELEGANT simulation at the location corresponding to Screen 14, where the CTR foil was inserted. An algorithm was used to reconstruct from the extracted $z$ coordinates the predicted autocorrelation function, including appropriate filtering of the frequency content due to diffraction, collection, and transport efficiency effects.

The simulated autocorrelation function was then subjected to the same fitting procedure that was used to extract $\sigma_{t}$ from the empirical interferograms, yielding values which produce the dashed curve in Fig. 6. These simulation results suggest that the observed compression and decompression results from a "folding over" of the longitudinal trace space due to the quadratic $T_{566}$ dependence in Eq. (2), where particles of both high and low energy begin to occupy the same longitudinal position within the bunch. This scenario is illustrated by the trace space plots in Fig. 7. The maximum compression [Fig. 7(b)] occurs at the sextupole field value where this folding over begins to change direction in $z$, corresponding to the point at which the second-order term $T_{566}$ changes sign. The discrepancy between theory and data near the fourth data point in Fig. 6 appears to be due to the sensitivity of the theoretical autocorrelation algorithm to the sharp spikes in the temporal distribution displayed in Figs. 7(c) and 7(d).

Although the temporal rms of the distribution is smaller in 7(c) than in 7(d), the spike is more pronounced in 7(c). That the physical data appears less sensitive to this effect may be related to additional frequency filtering produced by the interferometer in the short wavelength components of the spectrum. These effects may arise from the poor high frequency performances of the wire grid beam splitters in the Martin-Puplett device. Of course, one cannot rule out the possibility that the beam performance is not completely consistent with the predictions of simulations.

These sorts of uncertainties highlight the limited utility of CTR interferometry in this context and point to the need for more sophisticated measurements of the longitudinal phase space. The proposed diagnostic for performing longitudinal profile measurements in the Neptune experiment is a 9-cell deflecting mode cavity driven at an $X$-band frequency of $9.596 \mathrm{GHz}$, which is being designed in collaboration with the INFN Laboratori Nazionali di Frascati. When operating in the dipole mode $\mathrm{TM}_{110}$, such a cavity imparts to the beam a horizontal $(x)$ momentum kick that varies linearly with longitudinal position within the bunch. Consequently, the longitudinal distribution of the beam is deflected transversely and may be reconstructed from the
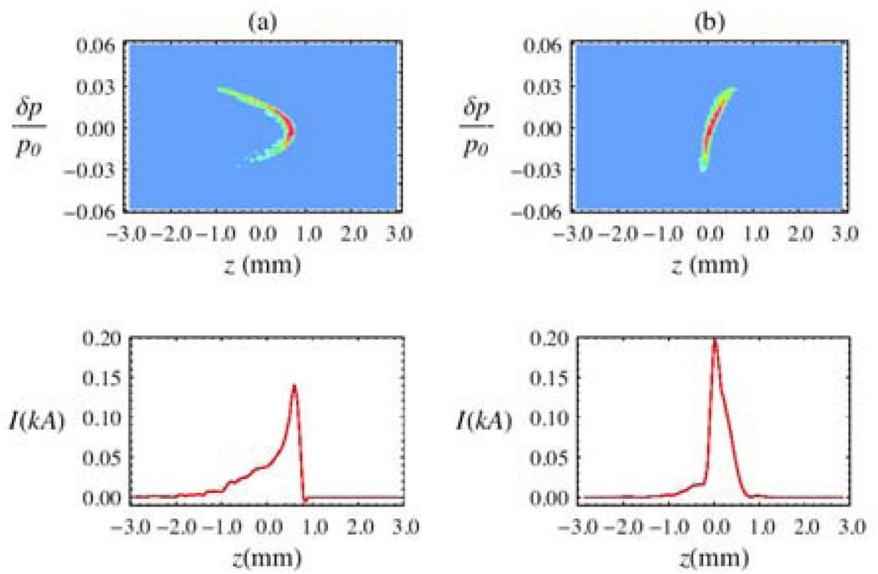

(c)
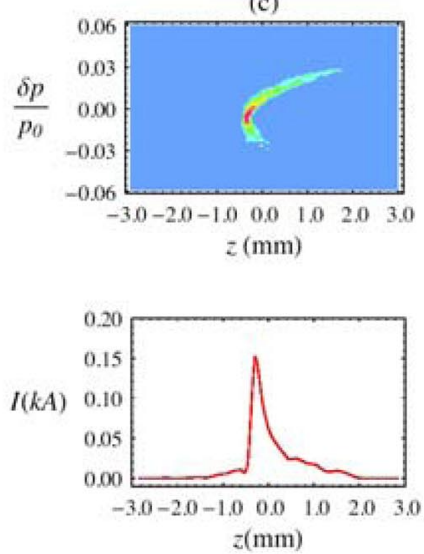

(d)
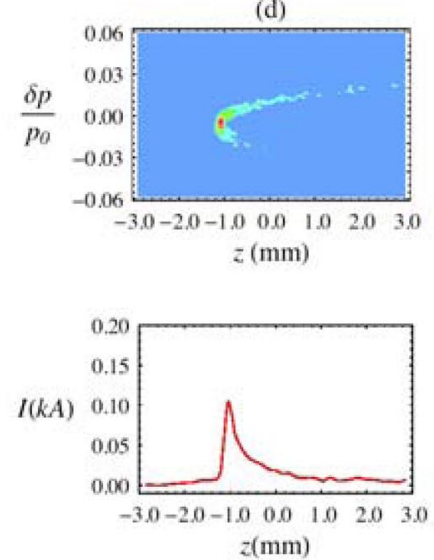

FIG. 7. (Color) The longitudinal phase space plots and density profiles obtained from the ELEGANT results corresponding to the sextupole values $\kappa=0,1094,1641$, and $2735 \mathrm{~m}^{-3}$, respectively, from the plot in Fig. 6, illustrating the progression of the phase space compression and decompression. The corresponding simulated $T_{566}$ values are $-2.11,0.02,1.08$, and $3.22 \mathrm{~m}$, respectively. 
image of the beam's cross section on a simple profile monitor downstream of the cavity. Thus one may obtain the beam's longitudinal profile, with an estimated resolution of $30 \mu \mathrm{m}$ (100 fs).

By combining the rf deflector with a magnetic dipole deflection along the orthogonal transverse $(y)$ axis, one obtains a complete reconstruction of the longitudinal trace space in both coordinate and momentum [20,21]. At the time of this writing, design work, using the commercial rf modeling code HFSS, has been completed, and a cold-test prototype has been constructed and tested. Completion and installation of a final cavity design are expected to occur in early 2005, providing the means to explore in much greater detail the complex dynamics contained in the results of Figs. 6 and 7.

\section{CONCLUSIONS}

We have examined the use of sextupole magnets to control second-order nonlinear chromatic effects and thereby optimize the compression and shaping of a relativistic electron bunch in a dispersionless translating section, or dogleg. For a beam of small transverse emittance, heuristic analytical arguments and simulation results indicate that the transformation of the distribution of the beam in $z$ is dominated by the first- and second-order transport matrix elements $R_{56}$ and $T_{566}$, which represent the linear and quadratic (in powers of momentum error) contributions to the longitudinal dispersion. Insertion of a pair of sextupole magnets with field strengths of equal magnitude $\kappa$ and opposite polarity provides a convenient method of manipulating the second-order term $T_{566}$, which is found to be linear in $\kappa$.

Simulation results using the beam transport codes PARMELA and ELEGANT, applying this type of correction to the Neptune and ORION beam lines, indicate that

longitudinal linearization aids in shaping the longitudinal trace space inside the dogleg compressor for creation of a ramped asymmetric current profile. This requires that the negative value of the $R_{56}$ be large enough to adequately compress the beam distribution. Additionally, for the example of the ORION beam line, Fig. 1(c), third-order effects are apparently a concern, due to the third-order longitudinal dispersion combined with a large energy spread. These effects are currently undergoing further study.

Recent experiments conducted on the UCLA-Neptune beam line provide evidence of the use of sextupole correction to manipulate both the second-order horizontal $\left(T_{166}\right)$ and longitudinal $\left(T_{566}\right)$ dispersion elements. The longitudinal dispersion studies were limited by the information which can be extracted from the CTR interferometry method utilized. Future planned experiments using a transverse deflecting mode cavity should yield detailed, highresolution longitudinal phase space measurements of the beam and provide a more complete understanding of the longitudinal phase space manipulations made possible by use of dogleg systems.

\section{ACKNOWLEDGMENTS}

The authors wish to thank E. Colby, D. Palmer, and D. Alesini for helpful discussions. This work was funded by the Department of Energy under Grant No. DE-FG0392ER40693.

\section{APPENDIX A: DERIVATION OF EQS. (3)-(6)}

First- and second-order transport matrices for different types of magnetic elements can be found in various references, including [6,22]. The first order matrices for a bend $B$ of bend angle $\theta$ and radius $\rho$, a thin-lens quadrupole $Q$ with focal length $f$, and a drift $D$ of length $l$ are as follows:

$$
\begin{gathered}
B(\theta, \rho)=\left(\begin{array}{ccccccc}
\cos \theta & \rho \sin \theta & 0 & 0 & 0 & \rho(1-\cos \theta) \\
-(\sin \theta) / \rho & \cos \theta & 0 & 0 & 0 & \sin \theta \\
0 & 0 & 1 & \rho \theta & 0 & 0 \\
0 & 0 & 0 & 1 & 0 & & 0 \\
-\sin \theta & \rho(\cos \theta-1) & 0 & 0 & 1 & \left(\frac{\rho \theta}{\gamma_{0}^{2}}-\rho \theta+\rho \sin \theta\right) \\
0 & 0 & 0 & 0 & 0 & \\
Q &
\end{array}\right) \\
Q(f)=\left(\begin{array}{cccccc}
1 & 0 & 0 & 0 & 0 & 0 \\
1 / f & 1 & 0 & 0 & 0 & 0 \\
0 & 0 & 1 & 0 & 0 & 0 \\
0 & 0 & -1 / f & 1 & 0 & 0 \\
0 & 0 & 0 & 0 & 1 & 0 \\
0 & 0 & 0 & 0 & 0 & 1
\end{array}\right) ;
\end{gathered}
$$

Let $Y$ represent the linear matrix for a combination of quadrupoles, sextupoles and drifts. The total first order matrix for a dogleg can then be written $R=B Y \tilde{B}$, where $B=B(\theta, \rho), \tilde{B}=B(-\theta,-\rho)$, and $Y$ has the form 


$$
Y=\left(\begin{array}{cccccc}
Y_{11} & Y_{12} & 0 & 0 & 0 & 0 \\
Y_{21} & Y_{22} & 0 & 0 & 0 & 0 \\
0 & 0 & Y_{33} & Y_{34} & 0 & 0 \\
0 & 0 & Y_{43} & Y_{44} & 0 & 0 \\
0 & 0 & 0 & 0 & 1 & Y_{56} \\
0 & 0 & 0 & 0 & 0 & 1
\end{array}\right) .
$$

The resultant horizontal dispersion function and its derivative (elements $R_{16}$ and $R_{26}$ of the total transport matrix) obtained by matrix multiplication are then given by

$$
\begin{aligned}
R_{16}= & \rho-\rho \cos \theta+\rho(\cos \theta-1)\left(Y_{11} \cos \theta+\rho Y_{21} \sin \theta\right) \\
& -\sin \theta\left(Y_{12} \cos \theta+\rho Y_{22} \sin \theta\right) \\
R_{26}= & \sin \theta+(\cos \theta-1)\left(\rho Y_{21} \cos \theta-Y_{11} \sin \theta\right) \\
& +\sin \theta\left(Y_{12} \sin \theta-\rho Y_{22} \cos \theta\right) / \rho
\end{aligned}
$$

The longitudinal dispersion element may then be written in terms of these functions as follows:

$$
R_{56}=Y_{56}+\frac{2 \rho \theta}{\gamma_{0}^{2}}+\left(2 \rho-R_{16}\right) \sin \theta+R_{26} \rho(1-\cos \theta) .
$$

Noting that $Y_{56}+2 \rho \theta / \gamma_{0}^{2}=\Delta s / \gamma_{0}^{2}$, where $\Delta s$ is the total path length, we see that Eq. (A5) reduces to Eq. (3) in the case where the quadrupoles are effectively utilized to eliminate linear horizontal dispersion $\left(R_{16}, R_{26} \rightarrow 0\right)$.

To obtain an analytical expression for the second-order longitudinal dispersion (element $T_{566}$ of the total transformation) we required the assistance of the commercial software package MATHEMATICA. Because of the cumbersome algebraic manipulations involved, we will merely outline the steps used to arrive at our results. Tabulations of the various second-order matrix elements may be found in $[6,22]$. For simplicity, we will represent the second-order counterparts to the linear matrices $B_{i j}, Y_{i j}$, and $\tilde{B}_{i j}$ by denoting them in component form using the same symbols but with three indices instead of two (i.e., $B_{i j k}, Y_{i j k}, \tilde{B}_{i j k}$ ). For $B_{i j k}$ and $\tilde{B}_{i j k}$ we use the analytical forms written in terms of $r$ and $\theta$ as given by [6,22]. For $Y_{i j k}$ we use a generic form equivalent to Eq. (A3), where we set to zero all elements which would naturally vanish for a system composed only of drifts, quads, and sextupoles. We then produce the total second-order matrix $T$ by successive multiplication of the matrices for the individual components, which we can write as $T_{i j k}=B_{i l}[Y \tilde{B}]_{l j k}+$ $B_{i l m}[Y \tilde{B}]_{l j}[Y \tilde{B}]_{m k}$, where $[Y \tilde{B}]_{i j k} \equiv Y_{i l} \tilde{B}_{l j k}+Y_{i l m} \tilde{B}_{l j} \tilde{B}_{m k}$ denotes the second-order matrix for the first two successive elements $\tilde{B}$ and $Y$ and there are implied sums on the repeated indices. This produces a set of equations for the elements $T_{i j k}$ in terms of $\rho, \theta, Y_{i j}$, and $Y_{i j k}$. Using these expressions, which are algebraically cumbersome and which we will therefore neglect to write out explicitly, the equation for the longitudinal dispersion element $T_{566}$ may be expressed as a linear combination of the expres- sions for the other matrix elements as follows:

$$
\begin{aligned}
T_{566}= & 4 \rho \sin ^{2}(\theta / 2) \cos (\theta / 2)+a_{16} R_{16}+a_{26} R_{26} \\
& +\sum_{W} a_{i 6 k} T_{i 6 k},
\end{aligned}
$$

where $W$ is the set of values in Eq. (5) and

$$
\begin{aligned}
a_{16} & =-\cos \theta \sin \theta, \quad a_{26}=\rho(1+2 \cos \theta) \sin ^{2}(\theta / 2), \\
a_{161} & =2 \rho \cos (\theta / 2) \sin ^{3}(\theta / 2), \quad a_{162}=-\frac{1}{2} \sin ^{2} \theta, \\
a_{166} & =\sin \theta, \quad a_{261}=-2 \rho^{2} \sin ^{4}(\theta / 2), \\
a_{262} & =2 \rho \cos (\theta / 2) \sin ^{3}(\theta / 2), \quad a_{266}=\rho(\cos \theta-1), \\
a_{561} & =-\rho \sin ^{2}(\theta / 2), \quad a_{562}=\frac{1}{2} \sin \theta .
\end{aligned}
$$

In the limit where $R_{16}, R_{26} \rightarrow 0$, we then find that Eq. (A6) reduces to Eq. (4).

To determine the dependence of $T_{566}$ on the sextupole field strength, let us assume that the sextupoles lie just inside the bends and are separated from each other only by quads and drifts. Although the same final result may be obtained without them, these assumptions will greatly simplify our calculation. Let the two sextupoles, of strengths $\kappa$ and $\alpha \kappa$, respectively, and of equal length $d$, be denoted by the symbols $S$ and $\tilde{S}$ and the intervening system of quads and drifts by $H$. We can then decompose the first- and second-order representations of $Y$ as $Y_{i j}=$ $S_{i k} H_{k l} \tilde{S}_{l j} \quad$ and $\quad Y_{i j k}=S_{i l}[H \tilde{S}]_{l j k}+S_{i l m}[H \tilde{S}]_{l j}[H \tilde{S}]_{m k}$, where $[H \tilde{S}]_{i j k} \equiv H_{i l} \tilde{S}_{l j k}+H_{i l m} \tilde{S}_{l j} \tilde{S}_{m k}$. Multiplying the linear matrices out explicitly and imposing the requirements

$$
R_{16}=0, \quad R_{26}=0, \quad \operatorname{det}\left(\begin{array}{ll}
H_{11} & H_{12} \\
H_{21} & H_{22}
\end{array}\right)=1,
$$

we arrive at the following conditions upon $H$ :

$$
\begin{aligned}
& H_{12}=-\left(1+H_{22}\right)(d+\rho \tan [\theta / 2]), \\
& H_{21}=\left(1-H_{22}\right) /(d+\rho \tan [\theta / 2]), \quad H_{11}=H_{22} .
\end{aligned}
$$

Applying these conditions in the calculation of the secondorder matrix, we arrive at the following result for element $T_{566}$ expressed in powers of $\kappa$ :

$$
\begin{aligned}
T_{566}= & 2 \sin ^{2}(\theta / 2)\left(A_{0} \rho \sin \theta+A_{+}+A_{-} \cos \theta\right) \\
& -\frac{d}{4} \sin ^{2} \frac{\theta}{2}\left[4\left|C_{0}\right|^{2}\left(2 \operatorname{Re} C_{0}-d \cos \frac{\theta}{2}\right) \sin \frac{\theta}{2}\right. \\
& \left.+8 \rho^{3} \cos \theta(1-\sin \theta)\right](1-\alpha) \kappa,
\end{aligned}
$$

where for the sake of compactness we have defined the functions 


$$
\begin{aligned}
A_{ \pm}= & H_{162}+H_{522}+d\left[H_{161}+H_{262}+H_{521}\right. \\
& \left.+d\left(H_{261}+H_{511}\right)-1\right] \pm \rho^{2}\left(H_{261}+H_{511}\right), \\
A_{0}= & 1+H_{161}+H_{262}+H_{521}+2 d\left(H_{261}+H_{511}\right) \\
& -\cos \theta, \quad C_{0}=d \cos \frac{\theta}{2}+(1+i) \rho \sin \frac{\theta}{2} .
\end{aligned}
$$

With the associations

$$
\begin{aligned}
A= & 2 \sin ^{2}(\theta / 2)\left(A_{0} \rho \sin \theta+A_{+}+A_{-} \cos \theta\right), \\
C= & \frac{d}{4} \sin ^{2} \frac{\theta}{2}\left[4 \sin \frac{\theta}{2}\left|C_{0}\right|^{2}\left(2 \operatorname{Re} C_{0}-d \cos \frac{\theta}{2}\right)\right. \\
& \left.+8 \rho^{3} \cos \theta(1-\sin \theta)\right],
\end{aligned}
$$

we find that Eq. (A10) takes the form of Eq. (6). The linear dependence on $\kappa$ is a reflection of the fact the second-order matrix elements for a sextupole are proportional to the field strength.

\section{APPENDIX B: DERIVATION OF EQ. (7)}

The full transformation in Eq. (1) represents the complete solution to the single-particle equations of motion, which constitute a Hamiltonian system. Therefore, to the extent to which the second-order transformation is an accurate description, it is Hamiltonian, and thus by Liouville's theorem the distribution function $f$ remains invariant under it. Consequently, $f\left(\boldsymbol{X}_{f}\right)=f\left(\boldsymbol{X}_{0}\right)$, where $\boldsymbol{X}_{f}$ and $\boldsymbol{X}_{0}$ are the final and initial trace space vectors, which are related in component form to second order by

$$
\left(X_{f}\right)_{i}=R_{i j}\left(X_{0}\right)_{j}+T_{i j k}\left(X_{0}\right)_{j}\left(X_{0}\right)_{k} \text {. }
$$

The matrix of second moments therefore transforms according to

$$
\begin{aligned}
\Sigma_{j k}= & \int\left[R_{j \ell}\left(\boldsymbol{X}_{0}\right)_{\ell}+T_{j \ell m}\left(\boldsymbol{X}_{0}\right)_{\ell}\left(\boldsymbol{X}_{0}\right)_{m}\right]\left[R_{k n}\left(\boldsymbol{X}_{0}\right)_{n}\right. \\
& \left.+T_{k n p}\left(\boldsymbol{X}_{0}\right)_{n}\left(\boldsymbol{X}_{0}\right)_{p}\right] f\left(\mathrm{X}_{0}\right) d^{6} X_{0},
\end{aligned}
$$

where the Jacobian of this transformation is

$$
J_{i j}=\frac{\partial\left(\boldsymbol{X}_{f}\right)_{i}}{\partial\left(\boldsymbol{X}_{0}\right)_{i}}=R_{i j}+\sum_{k} T_{i j k}\left(\boldsymbol{X}_{0}\right)_{k}\left(1+\delta_{j k}\right),
$$

with $\delta_{j k}$ representing the Kronecker delta. Writing Eq. (B2) in the bracket notation, we have

$$
\begin{aligned}
\Sigma_{j k}= & R_{j \ell} R_{k n}\left\langle\left(X_{0}\right)_{\ell}\left(X_{0}\right)_{n}\right\rangle+2 T_{k n p} R_{j \ell}\left\langle\left(X_{0}\right)_{\ell}\left(X_{0}\right)_{n}\left(X_{0}\right)_{p}\right\rangle \\
& +T_{j \ell m} T_{k n p}\left\langle\left(X_{0}\right)_{\ell}\left(X_{0}\right)_{m}\left(X_{0}\right)_{n}\left(X_{0}\right)_{p}\right\rangle,
\end{aligned}
$$

where there is an implied sum on repeated indices and $\langle\cdots\rangle \equiv \int \cdots f\left(\boldsymbol{X}_{0}\right) \operatorname{det} J d^{6} X_{0}$. Now assume the beam distribution function to be uncoupled between the three trace space planes, to have vanishing third moments, and unit Jacobian determinant. Then the upper left $2 \times 2$ submatrix of Eq. (B4) takes the form

$$
\Sigma_{x, f}=M_{x} \Sigma_{x, 0} M_{x}^{T}+\sigma_{\delta}^{2} d d^{T}+\left\langle\delta^{4}\right\rangle \boldsymbol{D} D^{T}+\Sigma_{\mathrm{geo}},
$$

where $\boldsymbol{d}, \boldsymbol{D}, M_{x}$, and $\Sigma_{x, 0}$ are the first- and second-order horizontal dispersion vectors, the $2 \times 2$ linear transport matrix for the $\left(x, x^{\prime}\right)$ trace space plane, and the initial $2 \times$ 2 matrix of second moments, respectively, as defined in Eq. (8), and $\Sigma_{\text {geo }}$ is the contribution from second-order geometrical terms. For a beam of small initial emittance and large energy spread, Eq. (B5) is dominated by the dispersion terms, and we can set $\Sigma_{\text {geo }} \ll\left\langle\delta^{4}\right\rangle \boldsymbol{D} \boldsymbol{D}^{T}$. Furthermore, if the beam distribution in the $z$ phase plane can be approximated by a rotated bi-Gaussian in $z$ and $\delta$, then $\left\langle\delta^{4}\right\rangle \cong 3 \sigma_{\delta}^{4}$. With these approximations, insertion of Eq. (B5) into the definition of the transverse emittance $\varepsilon_{x, f}=\sqrt{\operatorname{det} \Sigma_{x, f}}$ immediately produces Eq. (7).

\section{APPENDIX C: NONLINEAR COMPRESSION-VISA-I}

The VISA-I experiment [1] was a UCLA collaborative effort conducted from 1998 to 2001 for the purpose of studying SASE-FEL physics in the visible to near-infrared frequency range, using the $71 \mathrm{MeV}$ high-brightness beam provided by the Brookhaven ATF beam line. The presence on this beam line of a $15 \mathrm{~m}$ long dogleg section, shown in Fig. 1(a), combined with limitations in the transverse aperture presented a particular challenge from the perspective of beam transport. The beam line was operated in a configuration where the first- and second-order longitudinal dispersions were $R_{56}=-0.0045 \mathrm{~m}$ and $T_{566}=$ $-10.1 \mathrm{~m}$, respectively. The longitudinal transformation of Eq. (2) in the ATF dogleg section is dominated by the second-order $T_{566}$ contribution, and is therefore unsuitable for linear compression under ordinary conditions of operation. The gain of the SASE-FEL was found to be maximized when the beam was injected forward of crest in the accelerating cavity, giving it a negative momentum chirp and a $0.5 \%$ rms energy spread. This gain increase was due to a nonlinear compression of the beam in the dogleg section, resulting in a higher peak current. The pseudolinear compression was found to be produced by running the beam off-energy with a momentum $\tilde{p}$ differing from the central momentum $p_{0}$ of the design trajectory. Under this condition, the beam centroid follows the trajectory of an off-momentum particle whose momentum dispersion is $\Delta=\left(\tilde{p}-p_{0}\right) / p_{0}$. A particle with arbitrary momentum $p$ then has the momentum error $\tilde{\delta}=(p-\tilde{p}) / \tilde{p}$ relative to the central momentum of the beam and the momentum error $\delta=\left(p-p_{0}\right) / p_{0}$ relative to the design momentum for which the beam line is optimized. Applying the resultant coordinate transformation,

$$
\delta \rightarrow \frac{\tilde{p}}{p_{0}} \tilde{\delta}+\Delta,
$$

to Eq. (2), the longitudinal transport relative to the dis- 
(a)

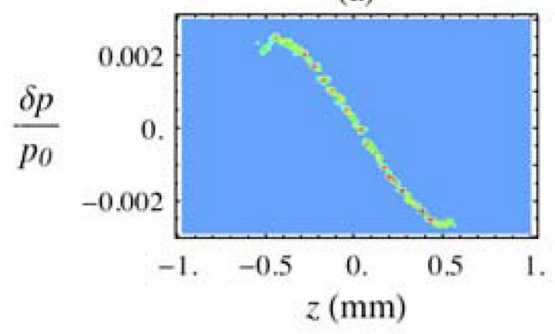

$I(A)$

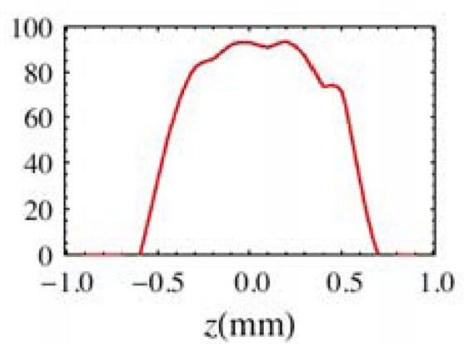

(b)
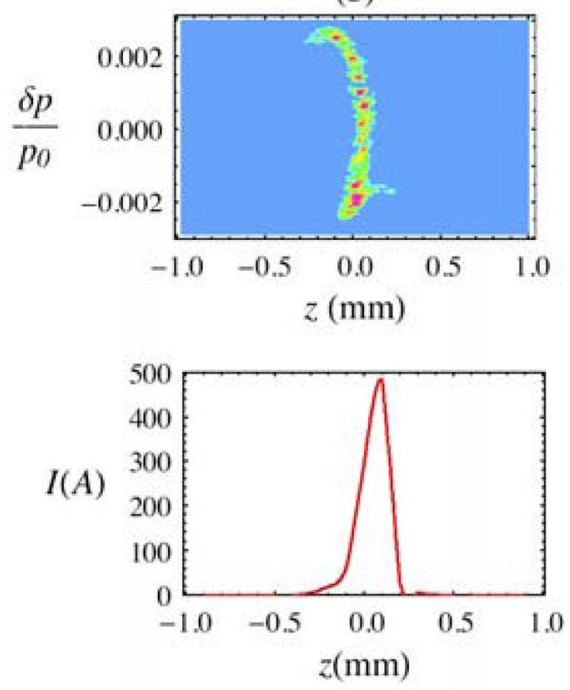

(c)

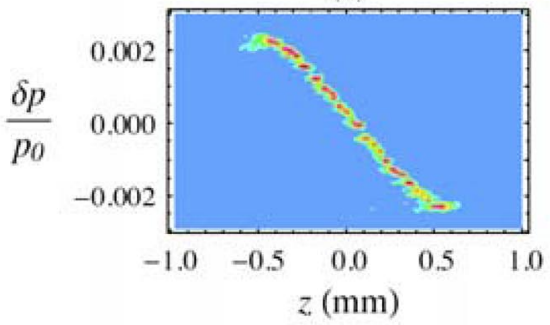

$I(A)$

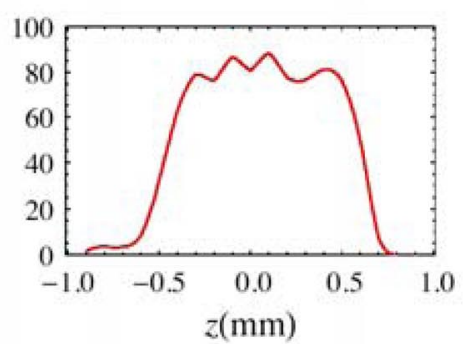

FIG. 8. (Color) ELEGANT simulations of the longitudinal trace space (upper plots) of the beam at ATF for VISA-I and corresponding current profiles (lower plots). The initial beam (a) shows a negative chirp, which undergoes a chicanelike compression (b). This compression is turned off (c) by the inclusion of sextupole correction.

placed momentum error $\tilde{\delta}$ is found to be given by

$$
z_{f}=z_{0}+\tilde{Q}_{5}+\tilde{R}_{56} \tilde{\delta}+\tilde{T}_{566} \tilde{\delta}^{2},
$$

where the transformation elements with respect to the new central momentum of the beam are (truncating third- and higher-order contributions)

$$
\begin{gathered}
\tilde{Q}_{5}=R_{56} \Delta+T_{566} \Delta^{2} ; \quad \tilde{R}_{56}=\frac{\tilde{p}}{p_{0}}\left(R_{56}+2 T_{566} \Delta\right) ; \\
\tilde{T}_{566}=\left(\frac{\tilde{p}}{p_{0}}\right)^{2} T_{566} .
\end{gathered}
$$

We may term the definitions given in Eq. (C3) the "effective" offset and momentum dispersion of the beam. They represent the values of $Q_{5}, R_{56}$, and $T_{566}$ that would be needed to obtain a similar transformation of the beam's longitudinal distribution if the beam were run onenergy. Using Eq. (C3) we find that operating the ATF beam line with a fractional energy offset of $\Delta=-0.76 \%$ produces a transformation that is dominated by an effective $\tilde{R}_{56}$ of $+0.18 \mathrm{~m}$, which is opposite in sign, and an order of magnitude larger in amplitude from $R_{56}$. Since $\tilde{R}_{56}$ is positive, the longitudinal compression which it produces is similar to that of a magnetic chicane and is therefore suitable for compressing a beam (as in the case of VISA-I) which has a negative (forward-of-crest) momentum chirp in $z$ and $\delta$. Although this scheme mimics the linear compression of a magnetic chicane, it is a primarily nonlinear effect, since the expression for the effective $\tilde{R}_{56}$ in Eq. (C3) is modified from its nominal value by the presence of the second-order $T_{566}$.

Sextupole correction would provide a convenient mechanism for changing the degree of compression pro- duced by this method, through its ability to manipulate the value of $T_{566}$, as discussed in Sec. III. This tunability is illustrated in Fig. 8, which shows ELEGANT simulations of longitudinal $(z-\delta)$ phase space distributions of a $71 \mathrm{MeV}$ beam on the VISA beam line. In Fig. 8(a) the phase space at the entrance of the dispersive section shows a beam with a strong momentum chirp. After being transported through the dispersive section with sextupole fields set to zero [Fig. 8(b)] the beam has been compressed by a factor of 4. With the sextupole fields in the simulation set to a field strength sufficient to force $T_{566}$ to vanish [Fig. 8(c)], the nonlinear compression is turned off and the final phase space resembles the initial distribution. The beam current is shown alongside each trace space plot.

The widening of the distribution in (c) as compared with (a) in Fig. 8 is due to the presence of nonlinear horizontalto-longitudinal coupling terms, such as $T_{561}, T_{562}$, and $T_{512}$, which we have neglected in Eq. (2). The resulting spread in $z$ therefore measures the degree of breakdown in the assumption of vanishing emittance. As this effect adversely affects the gain of the FEL radiation, minimization of the initial emittance is also a critical concern. In a related note, it should be emphasized that use of this nonlinear compression scheme requires running of the dogleg with nonzero dispersion, thus producing a larger effective emittance after transport.

\section{APPENDIX D: LINEARIZED BEAM TRANSPORT - VISA-II}

Part (c) of Fig. 9 illustrates the case where the elimination of $T_{566}$, combined with a small $R_{56}$, results in the approximate transformation $z_{f} \cong z_{0}$ whereby the beam is transported through the beam line with minimal perturba- 
(a)

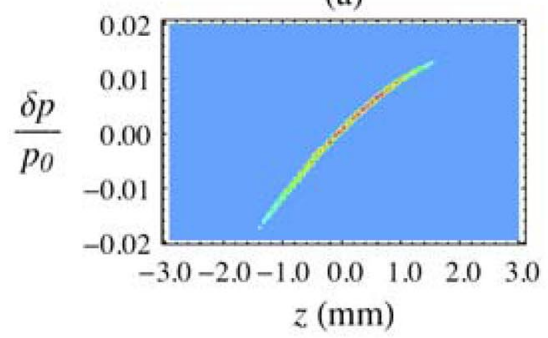

$I(A)$

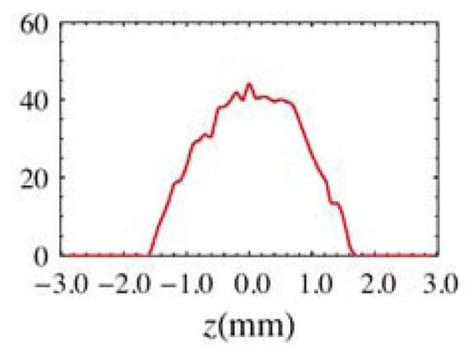

(b)

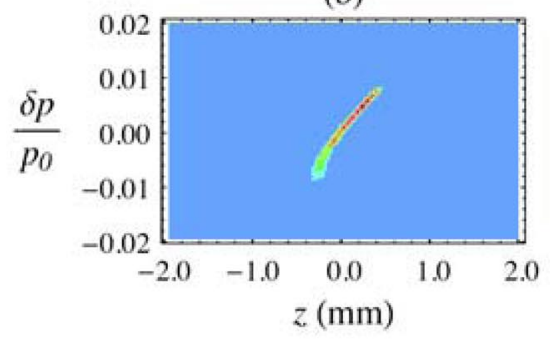

$I(A)$

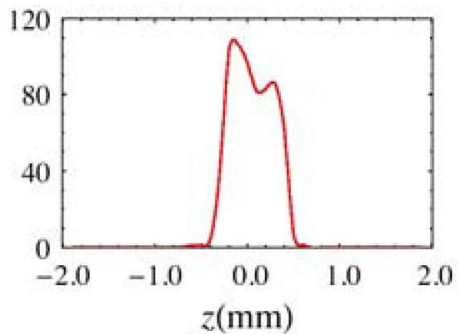

FIG. 9. (Color) ELEGANT simulation of the longitudinal trace space of the chirped beam at ATF for VISA-II, (a) before the dogleg section, and (b) after the dogleg section, including effects of collimators, which allow approximately $2 \%$ relative momentum spread to be transmitted.

tion of the longitudinal trace space distribution. This is useful in cases where it is critical to preserve the current profile of the beam. However, in the example of Fig. 8(c), the beam is run off-energy. In practice, this would result in dispersion mismatch and an offset of the beam centroid, which is generally undesirable. Consequently, a better course of action for this type of transport is to run onenergy in a configuration with a relatively small natural $R_{56}$ and with sextupole cancellation of the $T_{566}$.

The second stage of the VISA experiment [2] is a case in point, as included among its stated goals is the study of the time-frequency correlation of SASE-FEL radiation produced by injection of a positively chirped (back-of-crest) beam with a $2 \%$ rms energy spread into an undulator magnet. In this case, successful unperturbed transport of the approximately linear momentum chirp through the $15 \mathrm{~m}$ dogleg section on the ATF beam line requires control of the horizontal dispersion. This is accomplished through effective use of symmetrically placed quadrupoles, and the implementation of sextupole corrector magnets in regions of large dispersion. The results of this optimization can be seen in Fig. 9, which shows an ELEGANT simulation of the longitudinal trace space distribution 9(a) before and 9(b) after the dogleg section of the ATF beam line.

Here, the beam is run back of crest, giving it a negative momentum chirp and a 3\% full momentum spread. This momentum spread, which is larger than that which can be transmitted through a collimating region of the beam line after the initial bend, was chosen to give a certain value of the chirp $(d p / d z)$. This chirp leads to both compression in transport and the desired correlation between FEL radiation wavelength and longitudinal position in the pulse. Approximately $2 \%$ momentum spread may pass the colli- mators, however, corresponding to nearly a $40 \%$ loss in beam charge.

The components of the beam which are lost are ones which would yield low gain, and also are afflicted with undesirable nonlinear $z$ - $\delta$ correlations, as can be seen in Fig. 9(a). With the use of sextupoles, the nearly linear chirp in the transmitted component of the beam is successfully preserved, and enhanced in amplitude, during transit through the beam line. Note that there is a significant longitudinal decompression due to the dogleg's negative $R_{56}$, which in this configuration has a value of $-1.6 \mathrm{~cm}$. The peak current is enhanced by a factor of approximately 3 by this effect, as is the amplitude of the linear chirp after negotiation of the dogleg.

[1] A. Murokh et al., in Proceedings of the Particle Acclerator Conference, Chicago, IL, 2001 (IEEE, Piscataway, NJ, 2001), p. 2748.

[2] G. Andonian et al., in Proceedings of the Particle Accelerator Conference, Portland, OR, 2003 (IEEE, Piscataway, NJ, 2003), p. 944.

[3] R. J. England, P. Musumeci, R. Yoder, and J. B. Rosenzweig, in Proceedings of the Particle Accelerator Conference, Portland, OR, 2003 (Ref. [2]), p. 3258.

[4] R. J. Noble, E. R. Colby, D. T. Palmer, R. H. Siemann, D. Walz, R. Byer, C. Joshi, W. Mori, J. Rosenzweig, and T. Katsouleas, in Proceedings of the Particle Accelerator Conference, Portland, OR, 2003 (Ref. [2]), p. 1858.

[5] K. L. F. Bane, P. Chen, and P. B. Wilson, Stanford Linear Accelerator Center Technical Report No. SLAC-PUB3662, 1985.

[6] K. Brown, Stanford Linear Accelerator Center Technical Report No. SLAC-R-075, 1982. 
[7] M. Borland, Argonne National Laboratory Advanced Photon Source Technical Report No. LS-287, 2000.

[8] L. Young and J. Billen, Los Alamos National Laboratory Technical Report No. LA-UR-96-1835, 1996.

[9] C. E. Clayton, K. A. Marsh, A. Dyson, M. Everett, A. Lal, W. P. Leemans, R. Williams, and C. Joshi, Phys. Rev. Lett. 70, 37 (1993).

[10] K. Nakajima et al., Phys. Rev. Lett. 74, 4428 (1995).

[11] C. W. Siders, S.P. Le Blanc, D. Fisher, T. Tajima, and M. C. Downer, Phys. Rev. Lett. 76, 3570 (1996).

[12] F. Amiranoff et al., Phys. Rev. Lett. 81, 995 (1999).

[13] J. T. Seeman, IEEE Trans. Nucl. Sci. 30, 3180 (1983).

[14] K. L. F. Bane, P. B. Wilson, and T. Weiland, in Physics of High Energy Particle Accelerators, edited by M. Month, P.F. Dahl, and M. Dienes, AIP Conference Proceedings No. 127 (AIP, New York, 1985), p. 875.

[15] P. Chen, J. J. Su, J. M. Dawson, K. L. F. Bane, and P. B. Wilson, Phys. Rev. Lett. 56, 1252 (1986).
[16] J. G. Power, W. Gai, X. Sun, and A. Kanareykin, in Proceedings of the Particle Acclerator Conference, Chicago, IL, 2001 (Ref. [1]), pp. 114-116.

[17] S. G. Anderson, J. B. Rosenzweig, P. Musumeci, and M. C. Thompson, Phys. Rev. Lett. 91, 074803 (2003).

[18] D. H. Martin, in Infrared and Millimeter Waves, edited by K. J. Button (Academic, New York, 1982), Vol. 6, pp. 65148.

[19] A. Murokh, J. B. Rosenzweig, M. Hogan, H. Suk, G. Travish, and U. Happek, Nucl. Instrum. Methods Phys. Res., Sect. A 410, 452 (1998).

[20] G. A. Loew and O.H. Altenmueller, Stanford Linear Accelerator Center Technical Report No. SLAC -PUB$135,1965$.

[21] P. Emma et al., Stanford Linear Accelerator Center Technical Report No. LCLS TN-00-12, 2000.

[22] D.C. Carey, The Optics of Charged Particle Beams (Harwood Academic, New York, 1987), pp. 123-144. 